\title{
Human Milk: The Best Medicine for Vulnerable Babies
}

Heidi E. Karpen, MD

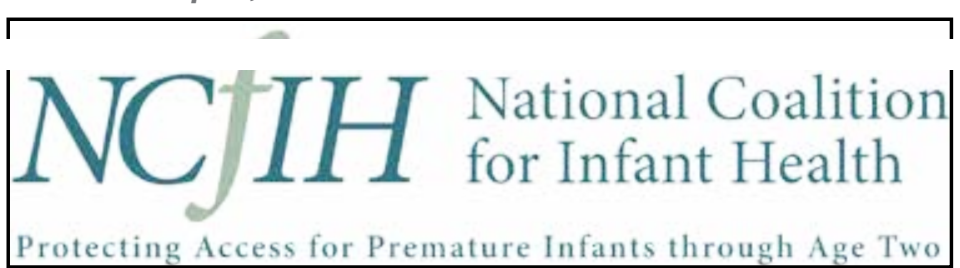

The National Coalition for Infant Health is a collaborative of more than 200 professional, clinical, community health, and family support organizations focused on improving the lives of premature infants through age two and their families. NCfIH's mission is to promote lifelong clinical, health, education, and supportive services needed by premature infants and their families. NCfIH prioritizes safety of this vulnerable population and access to approved therapies.

It fosters brain development. It passes on antibodies. It lowers the risk of infection. Human milk has long been known to offer many benefits to babies, especially to those born prematurely.

My colleagues and I are now working on a large, multi-hospital study that aims to shed new light on how human milk benefits one group of infants in particular - newborns with congenital gastrointestinal disorders. (1)

The babies in this study receive exclusive human milk diets. The milk is from their own mother or pasteurized donor human milk. All babies in the study also receive a human-milk-based fortifier to provide the additional calories and protein they need. The research team is comparing the outcomes of these babies to those who received infant formula as part of their diet. The expectation?
Infants receiving exclusively human milk will require IV nutrition for a shorter period of time. We are also comparing growth between the two groups of infants.
Positive results would reinforce a growing body of research on the topic of human milk. In prior research, babies with congenital gastrointestinal disorders spent about 20 fewer days in the hospital than infants fed formula primarily did. They also had few days of intravenous nutrition, fewer feeding problems, fewer infections, and less liver damage.

Congenital gastrointestinal disorders can occur when part of the intestine doesn't form correctly or when the intestines are outside the body through a hole in the abdomen. Newborns with these birth defects experience a delay in beginning to feed because of surgeries. Feeding intolerance and frequent feeding interruptions can also force them to rely on IV nutrition for long periods of time. These delays and problems in feeding their baby can lead new moms to decide not to breastfeed or pump milk.

A parent's decision to feed her baby human milk or formula is a personal one. It can also be a sensitive subject for new moms who 
may face unexpected challenges with milk supply, who are separated from their babies, lack support, or who are just beginning to comprehend what having a congenital gastrointestinal disorder will mean for their baby.

But data is proving that human milk might be the most effective medicine for babies with a congenital gut disorder. My goal is to help foster that education and offer support to new parents who decide that pumping and storing human milk until their baby is ready is right for them.

Human milk yields benefits to all babies, but especially those with a congenital gastrointestinal disorder.

\section{References:}

1. https://clinicaltrials.gov/ct2/show/NCT02567292

Heidi E. Karpen, MD, is a neonatologist at Emory University School of Medicine and a member of the National Coalition for Infant Health.

Disclosure: The author has no relevant disclosures.

\section{NT}

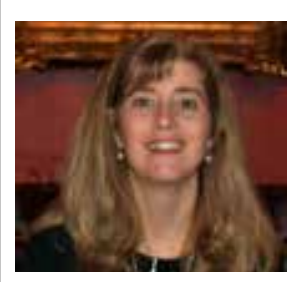

Heidi E. Karpen, MD

Associate Professor

SOM: Peds: Neonatology

Emory University School of Medicine

100 Woodruff Circle

Atlanta, GA 30322 USA

Email: heidi.karpen@emory.edu

\section{National Coalition for Infant Health Values (SANE)}

Safety. Premature infants are born vulnerable. Products, treatments and related public policies should prioritize these fragile infants' safety.

Access. Budget-driven health care policies should not preclude premature infants' access to preventative or necessary therapies.

Nutrition. Proper nutrition and full access to health care keep premature infants healthy after discharge from the NICU.

Equality. Prematurity and related vulnerabilities disproportionately impact minority and economically disadvantaged families. Restrictions on care and treatment should not worsen inherent disparities.

\section{OPIOIDS and NAS}

When reporting on mothers, babies,

and substance use

\section{LANGUAGE MATTERS}
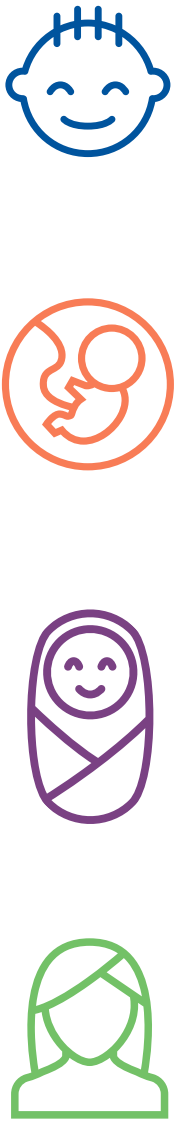

\section{My mother may have a SUD.}

She might be receiving Medication-Assisted Treatment (MAT). My NAS may be a side effect of her appropriate medical care. It is not evidence of abuse or mistreatment.

\section{My potential is limitless.}

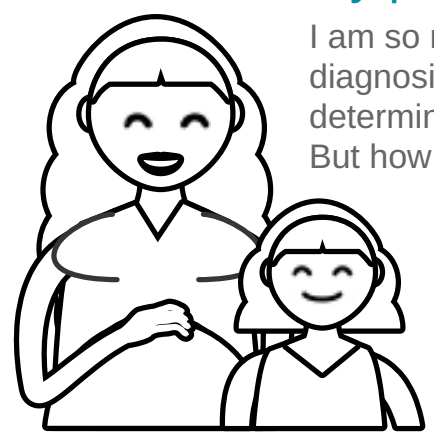

mo more than my NAS diagnosis. My drug exposure will not my long-term outcomes. ou treat me will. When you invest in my family's health and wellbeing by supporting Medicaid and Early Childhood Education you can expect that I will do as well as any of my peers!
Learn more about

Neonatal Abstinence Syndrome at www.nationalperinatal.org

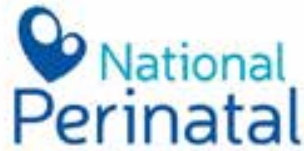

Association 